Nous estimons donc que le mode de cuisson, tel qu'il est actuellement pratiqué presque partout pour les pâtes alimentaires, ne convient nullement pour les pâtes au lactosérum, car il aboutit en fait à les ramener presque à l'état de pâtes ordinaires cuites (donc perte importante du point de vue nutritif comme du point de vue économique). Le mode de cuisson dit “ à l'étouffée " est assez délicat pour ces produits quant à la conduite de l'opération, si l'on veut éviter de les " brûler ». Il laisse, en outre, parfois un léger goût peu agréable avec certains lactosérums.

En conclusion, il nous apparaît donc, après essais pratiques, que seules, les pâtes au lactosérum du type " pâtes à potage " sont réellement intéressantes, puisque leur cuisson n'entraîne aucune élimination des substances nutritives complémentaires apportées et qu'elles donnent des potages à saveur réellement agréable.

\title{
REVUE
}

\section{COMPTE RENDU DU CONGRÈS D'AUTOMNE 1947 DE L'AMERICAN CHEMICAL SOCIETY}

\author{
par \\ G. GÉNIN
}

Avec la fin des hostilités, les Congrès de l'American Chemical Society ont retrouvé leur importance de jadis et le $112^{\text {e }}$ Congrès qui s'est tenu à New-York en septembre 1947 a attiré un nombre considérable de techniciens désireux de prendre connaissance des travaux accumulés au cours des années de guerre et qui, pour beaucoup d'entre eux, n'avaient pu être rendus publics, par suite des restrictions apportées à la publication de renseignements d'ordre technique.

Nous avons résumé dans ce qui suit un certain nombre de communications qui touchent plus ou moins directement l'industrie du lait et qui ont été présentées devant les diverses Sections du Congrès et plus particulièrement dans la Section de l'industrie des produits alimentaires.

\section{L'application des insecticides au bétail. Bishopp $F$. C.}

L'emploi des insecticides s'est considérablement développé au cours de ces dernières années. L'extrait de pyrèthre est resté l'agent le meilleur et le plus sûr pour la destruction des mouches qui s'attaquent au bétail et qui résident dans les étables, mais ce produit est coûteux et son effet n'est pas de longue durée. Le D. T. T. a une 
action plus lente, mais il a l'avantage de posséder une remarquable action résiduelle sur la plupart des espèces de mouches, aussi est-il employé sur une très grande échelle et est pulvérisé, non seulement à l'intérieur des étables, mais même directement sur le corps des animaux afin de les protéger contre les morsures des mouches.

Plus récemment, la découverte d'un nombre important de produits chlorés qui possèdent un pouvoir insecticide important, a augmenté les armes dont disposent les hommes pour mieux protéger le bétail contre l'action, non seulement des mouches, mais de tous les parasites. Il reste cependant à trouver encore des produits capables de mieux protéger les chevaux, ear les substances dont on dispose jusqu'à présent sont en général sans grande action sur les insectes qui s'attaquent au cheval.

Il reste encore beaucoup à faire dans le domaine de la préparation des insecticides, de leur mode d'emploi et de l'étude de leur action toxique.

\section{La recherche chimique et I'industrie laitière. Supplee G. (1)}

Le développement de l'industrie laitière repose en grande partie sur la mise, en œuvre de découvertes récentes qui ont résulté de recherches dans le domaine chimique ou dans le domaine biochimique. Les modes opératoires qui ont été employés au cours des nombreuses recherches dont ont fait l'objet le lait et ses dérivés, ont fait appel à des techniques les plus diverses empruntées aux domaines de la chimie, de la physique et de la biologie. On a pu dire que les progrès dans l'industrie du traitement et de la transformation du lait étaient liés directement aux progrès de la science biologique et de la science physique.

Malgré tous les progrès réalisés, le lait, en tant que produit de départ, pose encore pour l'habileté et l'intuition des chercheurs de très nombreux problèmes. Les recherches les plus prometteuses que l'on peut faire dans le domaine du lait appartiennent toutes plus ou moins à trois grandes catégories essentielles :

En premier lieu, ces recherches doivent porter sur la détermination quantitative et qualitative des nombreuses substances qui existent dans le complexe que constitue le lait. Ces recherches doivent également porter sur des propriétés physiques, comme par exemple, le potentiel d'oxydation-réduction, ou sur des propriétés colloïdales.

En second lieu, les travaux doivent être entrepris sur les produits couramment employés aujourd'hui en vue d'augmenter l'efficacité des méthodes que l'on utilise pour la préparation de ces produits et

(1) Cette conférence a été reproduite dans Le Lait, 1948, p. 279.289 (traduction G. Génin). 
en vue d'améliorer les qualités nutritives, organoleptiques et physiques de ces substances.

En troisième lieu, il faut chercher à développer l'utilisation du lait et de tous ses constituants dans la fabrication de substances nouvelles, intéressantes au point de vue alimentaire, au point de vue thérapeutique et au point de vue industriel. Le lait lorsqu'il est soumis à de telles transformations perd son identité et devient une véritable matière première de l'industrie chimique.

\section{Un produit nutritif, encore non identifié, du lait et des produits laitiers dégraissés. Hartman A. M. et Cary C. A.}

Les recherches ont montré que le lait, le lait écrémé, les poudres commerciales de lait écrémé et diverses qualités de fromage comme par exemple le fromage Cheddar, renferment un agent nutritif encore non identifié, que l'on ne trou ve ni dans la farine de froment, ni dans la farine de maïs, ni dans les tourteaux d'huile de soja ou d'huile de coton, ni dans la levure, ni dans le blane d'œuf coagulé, mais qui par contre existe dans certaines substances alimentaires, comme par exemple les feuilles de laitue, la viande de porc, la viande de bœuf, les extraits de foie, et qui joue un rôle important dans le traitement de l'anémie pernicieuse. La caséine contient cette substance, mais en proportion très variable, suivant la méthode que l'on a adoptée pour sa préparation. Certaines caséines en sont certainement privées, d'autres au contraire en sont riches et par exemple la caséine séparée du lait par dialyse est très riche en cette substance et favorise la croissance lorsqu'elle est ajoutée dans la proportion de $1 \%$ aux rations alimentaires.

Des rats nourris par des mères dont la nourriture a été volontairement appauvrie en cette nouvelle substance, souffrent également de cet appauvrissement et n'augmentent pas de poids normalement, même lorsqu'ils reçoivent comme nourriture des substances assurant normalement une croissance régulière. Par contre, dès que l'on ajoute à cette nourriture des faibles proportions de cette substance encore inconnue, la vitesse d'augmentation du poids peut devenir jusqu'à trois fois supérieure à celle d'animaux dans la ration desquels ne figurent pas de substance inconnue. On a pu préparer des extraits concentrés de cette substance qui s'est révélée active en dose journalière de quelques microgrammes.

\section{Les protéines immunisantes du lait et du colostrum de bovins. $S$ mith $E$. L.}

T. Sмгтн a montré, il y a quelques années, que le colostrum exerce une influence très favorable et protège par exemple les veaux nouveaux-nés contre les maladies infectieuses. 
Des travaux ont déjà été publiés sur l'isolement des protéines immunisantes du colostrum et $d u$ lait de bovin par E. L. SмrтH. Ces protéines immunisantes sont différentes par leurs propriétés physiques et par la composition de leurs amino-acides des autres protéines bien connues du lait comme la caséine ou la lactoglobuline. Par contre, elles ressemblent beaucoup aux protéines immunisantes que l'on trouve dans le sérum (globuline).

Une analyse électrophorétique a montré que le sérum des veaux nouveaux-nés ne contient pas de globuline se déplaçant lentement. Dès que le veau a absorbé du colostrum, le sérum renferme des quantités importantes de globuline avant la mobilité électrophorétique de la globuline immunisante du colostrum. Cette globuline se déplace plus rapidement que la globuline $\gamma$ de la vache adulte. Cette globuline pereiste longtemps chez le veau et le temps nécessaire pour que la concentration de cette globuline dans le sérum diminue à la moitié de sa valeur initiale est d'environ 20 jours. Par contre, la globuline $\gamma$ se décèle lorsque le veau atteint environ 50 jours.

Les facteurs qui influent sur la composition des protéines du lait de vache. McMeekin T. L., Della-Monica E. et Custer J. H.

Le lait commercial est un produit composite fourni par de nombreux animaux à différents moments de leur période de lactation. Les variations de la composition des protéines du lait peuvent intervenir sur leur emploi. Une étude a été effectuée sur des animaux pris individuellement, afin de déterminer l'influence de la période de lactation sur la composition des protéines du lait.

La caséine a été séparée du lait écrémé et purifiée par des précipitations iso-électriques. On a constaté par analyse électrophorétique que la composition de la caséine vis-à-vis de ses constituants et de la mobilité de ses constituants, est constante pendant la plus grande partie de la lactation. Cependant, à la fin de cette période et pendant plusieurs semaines avant la mise bas, la composition de la caséine varie et par exemple, la teneur en constituants $\beta$ diminue d'environ $25 \%$ à seulement $15 \%$. La caséine extraite du lait au cours de cette période contient moins de phosphore et d'azote et plus d'hydrates de carbone que la caséine moyenne.

Les protéines du sérum ont été analysées électrophorétiquement sans séparation préalable. Les proportions relatives de chacun des constituants restent constantes, jusqu'à environ 90 jours avant la mise bas. Après ce temps, la proportion de globuline $\gamma$ augmente progressivement jusqu'à la naissance du veau et devient ensuite normale 14 jours après la mise bas. 


\section{Distribution des dimensions des particules des protéines \\ du lait. Ford T. F., Ramsdell G. A. et Schaffer P. S.}

La caséine existe dans le lait de vache, sous forme d'un complexe easéinate de calcium-phosphate de calcium, dispersé sous forme de particules dont au moins 90 à $95 \%$ ont des vitesses de sédimentation qui correspondent à des poids moléculaires s'échelonnant de 16 millions à 256 millions. On ne trouve d'ailleurs pas entre ces limites toutes les valeurs possibles de poids moléculaire, mais uniquement des particules qui ont des poids moléculaires qui sont des multiples de 16 millions. Certaines de ces particules sont présentes en très fortes proportions, d'autres sont pratiquement absentes, certaines enfin existent en très faible proportion.

Il n'existe pas de différence au point de vue composition chimique élémentaire entre les petites et les grandes particules, mais cette analyse chimique élémentaire n'est pas capable de mettre en évidence les différences entre les groupes chimiques que l'on peut trouver dans les grandes particules et la preuve a été faite que d'importantes différences doivent exister entre ces groupes.

Quoique les particules de caséine dans le lait semblent être toutes constituées par des multiples d'un corps de poids moléculaire très élevé, cela ne signifie pas forcément que ce corps représente la molécule de caséine élémentaire. Dans le lait normal non traité, on trouve des particules de protéine précipitable par les acides et dont le poids moléculaire est de l'ordre de 500.000. Par différents traitements relativement doux, par exemple, par chauffage ou par action d'agents détersifs, les particules de caséine peuvent être scindées par étapes et on parvient à obtenir des produits d'un poids moléculaire d'environ 350.000 .

Les protéines solubles ne sont pas évidemment associées avee la caséine dans le lait. La proportion d'azote non caséinique dans le sérum du lait reste constante, après que la caséine a été séparée par centrifugation.

\section{L'enrichissement en tocophérol de la ration des vaches lai- tières. Hickman K., Swanson W.J. et Harris P. L.}

L'addition pendant une période de 20 mois d'un gramme de tocophérol à la ration journalière des vaches laitières, a entraîné une augmentation sensible de la production de graisse du lait dans le cas des races pures de Guernesey et de Suisse. L'action de cette addition se fait sentir rapidement, mais toutefois l'addition pendant trois semaines de tocophérol est encore insuffisante pour permettre de déterminer quelle est la ration la plus adéquate.

En procédant à une étude de la concentration en vitamine $\mathbf{E} d u$ lait fourni par des vaches qui recevaient une nourriture enrichie en 
tocophérol, on a pu établir qu'il faut plus de $0 \mathrm{gr}$. 35 de tocophérol par jour pour entraîner une augmentation sensible de la quantité de vitamine $\mathrm{E}$ sécrétée dans le lait.

Le lait de colostrum est riche en vitamine $\mathrm{E}$ et de ce fait, rapproché de ce que l'on sait que la teneur en vitamine $\mathbf{E}$ augmente dans le sang de la mère, montre l'importance de cette vitamine pour le veau nouveau-né. L'excrétion de vitamine $\mathrm{E}$ diminue d'environ $7 \mathrm{mgr}$. par litre de colostrum à environ $1 \mathrm{mgr}$. par litre, après la première semaine de lactation.

\section{L'emploi des dérivés de l'acide lactique comme plastifiants. Les diglycols bis-carbonates des esters lactiques. Rehberg C. E., Dixon M. B. et Fisher C. H.}

On a préparé 23 diglycols bis-carbonates d'esters lactiques en faisant réagir des diglycols bis-chloroformiates sur des esters lactiques. Ces esters avaient des poids moléculaires s'échelonnant entre 366 et 627 et des points d'ébullition sous un millimètre de mercure variant de 200 à environ $275^{\circ}$.

Tous les esters ainsi obtenus sont compatibles avec les chloroacétates de polyvinyles ou avec l'acétate de cellulose et plusieurs d'entre eux sont compatibles avec l'éthylcellulose. On a déterminé la résistance à la traction, le module pour un allongement de $100 \%$, l'allongement à la rupture et la fragilité pour différentes résines vinyliques plastifiées par ces esters, en même temps qu'on déterminait la tension de vapeur, la viscosité, la solubilité dans l'eau, la stabilité vis-à-vis de l'eau bouillante et l'acidité libre de ces différents plastifiants.

Ces esters pouvant se préparer avec un rendement satisfaisant, en partant de différents produits aujourd'hui peu coûteux et d'un approvisionnement facile et plusieurs d'entre eux se comparant très favorablement au phtalate de dioctyle comme plastifiant des résines vinyliques, il est probable qu'ils seront appelés à jouer un rôle important dans l'industrie des plastiques.

\section{Les dérivés de l'acide lactique comme plastifiant. Esters des acides lactiques polymérisés. Filachione E. M., Costello E. J. et Fisher C. H.}

On a préparé un certain nombre de dérivés acylés des polylactates d'alcoyle et on a étudié l'emploi de ces produits comme plastifiant des polymères de ehlorure de vinyle. Les polylactates d'alcolyle se préparent en partant de lactate de méthyle, d'éthyle ou de butyle, en chauffant ces lactates en présence d'un catalyseur constitué par un acide minéral et en distillant l'alcool correspondant, e'est-à-dire alcool méthylique, éthylique ou butylique. L'importance 
de la polymérisation est déterminée par la quantité d'alcool éliminée par distillation et par les conditions de l'expérience. Les polymères de faible poids moléculaire s'obtiennent facilement en chauffant les lactates entre 100 et $175^{\circ}$ et en éliminant l'alcool sous pression réduite.

Les polylactates peuvent également se préparer par alcoolyse du lactate de méthyle avec un alcool supérieur et élimination par distillation de l'alcool méthylique. En utilisant environ trois molécules de lactate de méthyle pour une molécule d'alcool supérieur, on obtient des polymères de faible poids moléculaire. Les polylactates ainsi préparés sont probablement des mélanges de polylactates de méthyle et de polylactates d'alcoyles supérieurs.

Une troisième méthode de préparation des polylactates d'alcoyle qui convient partieulièrement bien à la préparation des polylactates d'alcools supérieurs, repose sur l'estérification d'un mélange contenant trois ou un plus grand nombre de molécules d'acide lactique, une molécule d'alcool en présence d'un catalyseur constitué par un acide minéral. L'eau est éliminée en effectuant l'estérification dans le vide, ou en utilisant un agent d'entraînement. Les polylactates de $\beta$-butoxyéthanol, de n-octyl et d'alcool 2-éthylhexyle, peuvent se préparer par cette méthode.

On peut alors procéder à l'acylation des polylactates d'alcoyle en utilisant un réactif tel que l'anhydride acétique, l'anhydride propionique, le chlorure de benzoyle, le chlorure de lauroyle, le chlorure de 2-éthylhexoyle et le chloroformiate de diglycol. Les produits obtenus sont des mélanges d'esters d'acide lactique monomère, dimère, trimère et polymère. Ils ne sont pas distillés, mais isolés sous forme de mélange après lavage et traitement par un charbon activé. Les produits que l'on obtient ainsi sont presque incolores, ils se présentent sous la forme de liquides visqueux de point d'ébullition élevé.

Ces esters sont généralement compatibles avec l'éthylcellulose et avec le copolymère de $95 \%$ de chlorure de vinyle et de $5 \%$ d'acétate de vinyle. Ils ne sont généralement pas compatibles avec l'acétate de cellulose.

\section{Les polymères de l'acide lactique comme constituants des résines et vernis synthétiques. Watson $P$. $D$.}

Le but de cette étude a été de décrire les différents polymères modifiés obtenus par condensation de l'acide lactique qui ont été mis au point dans les Dairy Research Laboratories et qui présentent un certain intérêt pour l'industrie des vernis. On a estimé que le sérum fourni chaque année en tant que sous-produit de la fabrication de la caséine et du fromage, pourrait à lui seul assurer la fabri- 
cation annuelle d'environ 400 millions de pounds d'acide lactique. Le manque d'étain et de produits nécessaires à la fabrication des vernis dont on a souffert pendant la guerre montre l'intérêt de l'utilisation de l'acide lactique dans ce domaine.

Le caractère bifonctionnel de l'acide lactique permet à ce produit de former des polymères par self-estérification et ces produits à leur tour peuvent donner naissance à des résines lorsqu'on les fait réagir avec des agents contenant des groupes fonctionnels tels que aldéhydes, esters, hydrates de carbone, huiles grasses et sels métalliques. Les acides polylactyliques s'obtiennent par chauffage et par déshydratation de l'acide lactique, de préférence sous pression réduite ou par élimination de l'eau.

Les plus utiles de ces résines sont les polymères d'acides polylactyliques modifiés par les huiles grasses qui forment des pellicules résistantes, flexibles et insensibles à l'action de l'eau. Ces résines se préparent en faisant réagir l'acide polylactylique avec des huiles telles que l'huile de ricin, l'huile de lin ou les huiles siccatives modifiées à des températures comprises entre environ 265 et $280^{\circ}$, jusqu'à ce que l'on obtienne une résine élastique et brune. On a méliore les qualités de ces produits, en ajoutant de petites quantités d'acide fumarique, d'anhydride maléique, d'hydrocarbures oléfiniques, d'hydrocarbures cycloparaffiniques extraits du goudron de houille et des résines phénoliques solubles dans l'huile. De très intéressants résultats ont été déjà enregistrés par les fabriques de vernis qui ont étudié l'emploi de ces substances.

Une autre eatégorie nouvelle de résines est constituée par les polylactyllactates métalliques provenant presque entièrement de l'acide lactique et que l'on peut employer comme vernis protecteur et décoratif. Ces polymères s'obtiennent en faisant réagir un acide polylactylique avec des sels métalliques solubles tels que les sels d'aluminium, de chrome, de cobalt, de cuivre, de fer, de manganèse, d'étain ou des combinaisons de ces sels. Les chaînes linéaires d'acide polylactylique sont réunies à leurs extrémités carboxyles par les atomes métalliques multivalents, avec formation de structure à trois dimensions. Ces résines peuvent être améliorées par fractionnement.

Le filage continu des filaments de caséine. Petterson $R$. $F$., McDowell R. L. et Hoover S. R.

Il a été possible de mettre au point le filage continu des filaments de caséine, grâce à un procédé de durcissement accéléré qui rend possible l'utilisation dans cette technique du filage par pot centrifuge, utilisé dans l'industrie de la rayonne. Le filament que l'on obtient est supérieur par ses qualités mécaniques à la soie artifi- 
cielle. Sa ténacité à la température de $25^{\circ} \mathrm{C}$. et pour une humidité relative de $65 \%$ est d'environ $1 \mathrm{gr}$. 2 par denier et sa résistance lorsqu'il est humide est d'environ $0 \mathrm{gr}$. 55 par denier. L'allongement à la rupture est d'environ $50 \%$ à l'état sec ou à l'état humide.

L'amélioration des qualités mécaniques à l'état sec ou à l'état humide, par rapport à celle des filaments ordinaires, est obtenue en faisant subir au fil un allongement dans des solutions chaudes de sels et de formaldéhyde. La teneur en formaldéhyde du filament est d'environ $2 \%$, mais $0,5 \%$ seulement de ces produits sont liés d'une façon suffisante au filament pour résister au lavage.

Quoique la force centrifuge s'exerçant sur le filament humide tende à plaquer et à coller les filaments entre eux et à diminuer leur flexibilité, on peut compléter l'action du durcissement par la formaldéhyde par une réaction rapide avec des sels d'aluminium. Après avoir été emmagasinée pour que la réaction avec la formaldéhyde se termine, la fibre est lavée, séchée, puis acétylée pour augmenter sa résistance à l'ébullition. Pour que cette dernière opération se fasse convenablement, il est important de régler avec soin la teneur en humidité de la fibre, car l'acide acétique produit au cours de la réaction tend à dégrader la fibre.

La réaction avec les silicones permet également d'améliorer les qualités de la fibre, non seulement à l'état humide, mais également à l'état sec.

\section{Absorption d'eau par les protéines. - II. Groupes aminés libres et hystérésis dans la caséine. Mellon E. F., Korn A. $H$. et Hoover S. $R$.}

On a utilisé un certain nombre de dérivés benzoylés de la caséine, présentant des teneurs bien déterminées en groupes aminés, afin de déterminer l'absorption en phase vapeur par les groupes aminés des protéines.

Les essais de désorption ont montré que pour ehaque dérivé examiné, il existe un isotherme de désorption caractéristique que l'on obtient, quelle que soit l'humidité relative avec laquelle on opère. L'hystérésis au cours de ces phénomènes est importante, mais indépendante de la teneur en groupes aminés du dérivé.

Lorsque l'on trace la courbe représentant la désorption en fonction de l'absorption, on obtient une ligne droite pour toutes les humidités relatives comprises entre 5 et $50 \%$. Les phénomènes ainsi observés ont été interprétés en admettant qu'une partie tout au moins des phénomènes d'hystérésis ne dépend pas de phénomènes de condensation capillaire. 